\title{
Nonstoichiometric Effects in the Leakage Current and Electrical Properties of Bismuth Ferrite Ceramics
}

\author{
Jeong Wook Woo*, SeungBong Baek*, Tae Kwon Song*, Myang Hwan Lee*, Jamil Ur Rahman****, \\ Won-Jeong Kim****, Yeon Soo Sung*****, Myong-Ho Kim**t, and Soonil Lee***t \\ ${ }^{*}$ School of Advanced Materials Engineering, Changwon National University, Changwon 51440, Korea \\ **Energy and Environmental Materials Division, Korea Institute of Ceramic Engineering and Technology, Jinju 52851, Korea \\ ***Department of Physics, Changwon National University, Changwon 51140, Korea \\ ****Department of Materials and Engineering, Pohang University of Science and Technology, Pohang 37673, Korea
}

(Received May 24, 2017; Revised June 22, 2017; Accepted June 22, 2017)

\begin{abstract}
To understand the defect chemistry of multiferroic $\mathrm{BiFeO}_{3}$-based systems, we synthesized nonstoichiometric $\mathrm{Bi}_{1+\mathrm{x}} \mathrm{FeO}_{3 \pm \delta}$ ceramics by conventional solid-state reaction method and studied their structural, dielectric and high-temperature charge transport properties. Incorporation of an excess amount of $\mathrm{Bi}_{2} \mathrm{O}_{3}$ lowered the Bi deficiency in $\mathrm{BiFeO}_{3}$. Polarization versus electric field (P-E) hysteresis loop and dielectric properties were found to be improved by the $\mathrm{Bi}_{2} \mathrm{O}_{3}$ addition. To better understand the defect effects on the multiferroic properties, the high temperature equilibrium electrical conductivity was measured under various oxygen partial pressures $\left(\mathrm{O}_{2}\right.$ 's). The charge transport behavior was also examined through thermopower measurement. It was found that the oxygen vacancies contribute to high ionic conduction, showing $p \mathrm{O}_{2}$ independency, and the electronic carrier is electron ( $n$-type) in air and $\mathrm{Ar}$ gas atmospheres.
\end{abstract}

Key words : Bismuth ferrite, Ferroelectric, Electrical conductivity, Defect, Nonstoichiometry

\section{Introduction}

$\mathbf{I}_{\mathrm{s}}^{\mathrm{n}}$ February 2003, the Restriction of Hazardous Substances Directive (RoHS) was enacted in Europe and on July 1, 2006, a regulation was enacted restricting the use of lead, cadmium, mercury, hexavalent chrome, phthalates, and brominated flame retardants (PBBs, PBDEs) in electronic products and devices. After such enactments, the usage of lead was regulated, resulting in active research on lead-free (Pb-free) piezoelectric ceramics to replace PZT based materials. Representatively, studies on alkali metal based $\left(\mathrm{Na}_{0.5} \mathrm{~K}_{0.5}\right) \mathrm{NbO}_{3}(\mathrm{NKN})$, bismuth based $\left(\mathrm{Bi}_{0.5} \mathrm{Na}_{0.5}\right) \mathrm{TiO}_{3}$ (BNT) and $\mathrm{BiFeO}_{3}(\mathrm{BFO})$ compositions are widely being carried out. ${ }^{1-4)} \mathrm{BFO}$ has a rhombohedral (R3c) structure and exhibits multiferroic properties, in which antiferromagnetic and ferroelectric properties coexist at room temperature. ${ }^{5)}$ Also, BFO has significant advantages of a high Curie temperature $\left(\mathrm{T}_{\mathrm{C}}\right)$ of $827^{\circ} \mathrm{C}$ and high Neel temperature $\left(\mathrm{T}_{\mathrm{N}}\right)$ of $367^{\circ} \mathrm{C}{ }^{6)}$ According to the literature, $\mathrm{BFO}$ shows a value of $90-100 \mu \mathrm{C} / \mathrm{cm}^{2}$ when the spontaneous polarization $\left(\mathrm{P}_{\mathrm{S}}\right)$ value is orientated in the [111] direction. ${ }^{7)}$ Despite having

\footnotetext{
${ }^{\dagger}$ Corresponding author: Myong-Ho Kim

E-mail : mhkim@changwon.ac.kr

Tel : +82-55-213-3711 Fax : +82-55-262-6486

"Corresponding author : Soonil Lee

E-mail : leesoonil@gmail.com

Tel : +82-55-792-2567 Fax : +82-55-792-2580
}

such excellent properties, BFO is known to have limitations in applicability due to its high leakage current. The causes of the leakage current of $\mathrm{BFO}$ have been reported to be the combined effect of $\mathrm{Bi}$ volatility in the high temperature sintering process and defects of oxygen vacancies due to the transition metal Fe. ${ }^{8,9)}$ Also, recent reports have shown the effects of secondary phases of $\mathrm{BFO}$ like $\mathrm{Bi}_{25} \mathrm{FeO}_{39}$ and $\mathrm{Bi}_{2} \mathrm{Fe}_{4} \mathrm{O}_{9}$, which appear in a specific temperature range (447 - $\left.767^{\circ} \mathrm{C}\right) .{ }^{10,11)}$ However, sufficient context is lacking to clearly explain the fundamental cause behind the leakage current due to the significant variation of material properties that result from $\mathrm{Bi}$ volatility and $\mathrm{Fe}$ transition. In this study, to measure the ferroelectric P-E hysteresis loop, dielectric constant, and dielectric loss at room temperature, and to investigate the leakage current mechanism of BFO, the amount of $\mathrm{Bi}$ vulnerable to high temperatures was controlled and nonstoichiometric BFO ceramics of $\mathrm{Bi}_{1+\mathrm{x}} \mathrm{FeO}_{3 \pm \delta}$ were fabricated. Additionally, so that the mechanism behind the main defects and charge transport, which cause the leakage current, could be understood, and in order to identify defects in a specific range, the electrical conductivity and Seebeck coefficient were measured at high temperature with various temperatures and oxygen partial pressures $\left(\mathrm{pO}_{2}\right)$.

\section{Experimental Procedures}

In the case of $\mathrm{BFO}$, it is known that fabricating a single phase is difficult due to the volatility and transition issues 
of the constituent elements. Single phase BFO can be synthesized when the constituent elements $\mathrm{Bi}_{2} \mathrm{O}_{3}$ and $\mathrm{Fe}_{2} \mathrm{O}_{3}$ have a stoichiometric ratio of exactly $1: 1$. However, since long-term exposure to high temperature is necessary for the ceramic sintering in the fabrication process, it is difficult to evade the temperature range $\left(447-767^{\circ} \mathrm{C}\right)$ for secondary phase production. In order to overcome this issue, the powder produced after heat treatment was reacted with nitric acid to remove the secondary phase; sol-gel or hydrothermal methods were used to reduce the particle size; or spark plasma sintering (SPS) and rapid sintering were used to reduce the exposure time to the high sintering temperature. ${ }^{12,13)}$ However, the above experiment involves cost and environmental issues because acids are used, like nitric acid for the fabrication. Thus, in this study, the general solidstate reaction method was used to prevent the production of the secondary phase after sintering; also, the quenching method was used to rapidly cool to room temperature for BFO ceramic fabrication.

\subsection{Powder Synthesis and Specimen Preparation}

Starting materials $\mathrm{Bi}_{2} \mathrm{O}_{3}$ (Alpha acer 99.99\%) and $\mathrm{Fe}_{2} \mathrm{O}_{3}$ (Alpha acer 99.99\%), of high purity, were used to fabricate BFO. The mol\% values of the starting materials were calculated and weighted according to $\mathrm{Bi}_{1+\mathrm{x}} \mathrm{FeO}_{3 \pm \delta}(\mathrm{x}=0,0.05)$. Then, ethanol was used as the solvent, along with the $\mathrm{ZrO}_{2}$ balls, to carry out ball milling for $24 \mathrm{~h}$ to improve the homogeneity of mixed powder. Afterwards, each specimen was denominated as 0BFO $(\mathrm{x}=0)$ and $5 \mathrm{BFO}(\mathrm{x}=0.05)$. The mixed powder was dried using a dryer and the dried material was heat treated at $700^{\circ} \mathrm{C}$ under ambient air conditions for $2 \mathrm{~h}$. After the heat treatment, the material was ground in a mortar to improve the powder homogeneity and PVA (polyvinylalcohol) was added to enhance the integration between the powders. The homogeneity of the PVA-added powder was further improved through sieving and the ceramic was fabricated using a uniaxial press.

\subsection{Sintering Process}

Microstructure observation of the BFO ceramic fabricated using the general sintering method (Fig. 1(a)), which performs sintering by raising the temperature to the final sintering temperature in one step, revealed nonuniform grains and voids here and there. ${ }^{14,15)}$ Such voids were observable due to numerous variables including the sintering temperature, sintering time, and heating rate. The two-step sintering method was used to effectively improve such problems in the sintering process. ${ }^{16)}$ In order to resolve the reaction issue during high temperature sintering between the $\mathrm{Al}_{2} \mathrm{O}_{3}$ and $\mathrm{Bi}$ ion of the $\mathrm{BFO}$, a sufficient amount of $\mathrm{BFO}$ powder was applied on an alumina plate to prevent direct reaction between the $\mathrm{BFO}$ specimen and $\mathrm{Al}_{2} \mathrm{O}_{3}$. In addition, in order to prevent $\mathrm{Bi}$ ion volatility, which can occur during the high temperature sintering process, sintering was carried out after sufficient application of the BFO powder on the BFO specimen. Also, the temperature of $300^{\circ} \mathrm{C}$ was maintained for $1 \mathrm{~h}$ to remove the PVA in the specimen; then, the temperature was rapidly raised at a rate of $10^{\circ} \mathrm{C} / \mathrm{min}$ to reduce defects that can occur in the secondary phase generation range of $447-767^{\circ} \mathrm{C}$. Then, after maintaining a temperature of $750^{\circ} \mathrm{C}$ for 30 minutes, the heating rate was decreased to $2^{\circ} \mathrm{C} / \mathrm{min}$ in order to reduce problems like voids within the ceramic; heating was continued until the final sintering temperature of $820^{\circ} \mathrm{C}$. Finally, the temperature of $820^{\circ} \mathrm{C}$ was maintained for 10 minutes; this was followed by air quenching for rapid cooling until room temperature was reached, so that secondary phase generation during cooling can be prevented. Fig. 1(b) shows the overall two-step sintering process. The structure and crystallinity of the produced specimen were analyzed through XRD (X-ray Diffractometer, Miniflex II, Rigaku); SEM (Scanning Electron Microscope, SEM, JSM-6510) was carried out for microstructural observation.

\subsection{Electrical Conductivity Measurement}

After cutting the $\mathrm{Bi}_{1+\mathrm{x}} \mathrm{FeO}_{3 \pm \delta}(\mathrm{x}=0,0.05)$ specimen to dimensions of $2 \times 2 \times 15 \mathrm{~mm}^{3}$, sputtering was used to apply platinum electrodes at regular intervals on the specimen surface. Each of the 4 platinum electrodes was connected to platinum wires and the electrical conductivity was measured for the temperature range of $650-750^{\circ} \mathrm{C}$. To eliminate the issue of non-ohmic contact, the electrical conductivity was measured using the 4-probe method. The following equation was used to convert the resistance obtained from the specimen measurement to the electrical conductivity.
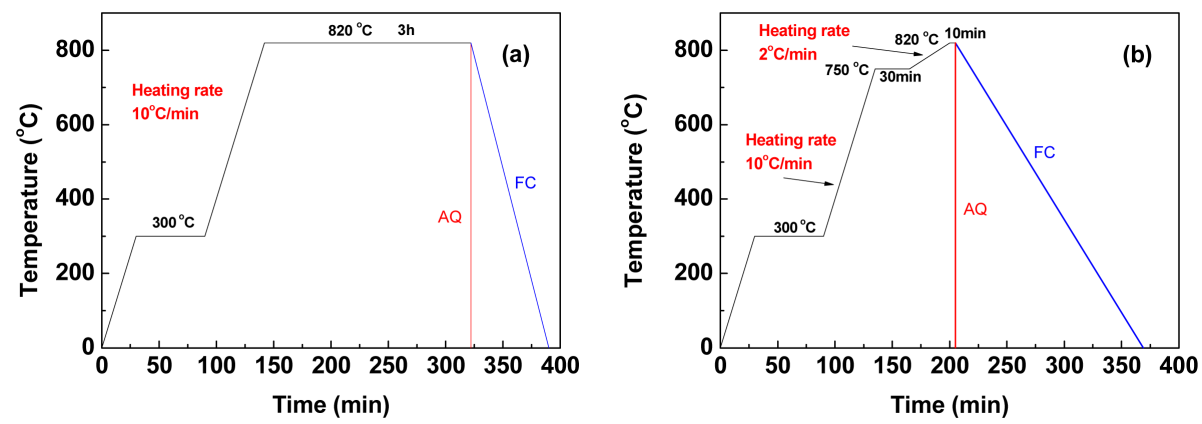

Fig. 1. Conditions for (a) conventional sintering and (b) two-step sintering process; air-quenched (AQ) and furnace-cooled (FC). 


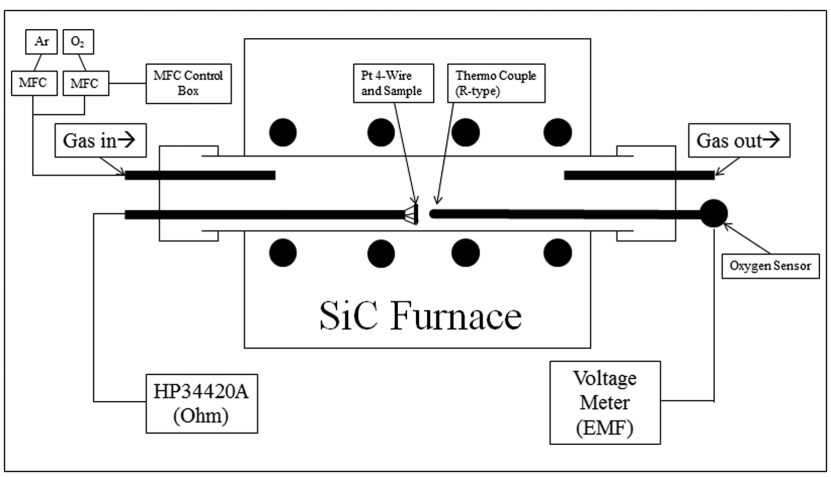

Fig. 2. Schematic diagram for high temperature equilibrium conductivity measurement system.

$$
\sigma=\frac{l}{R \times A}
$$

Here, $R$ is the resistance $[\Omega], A$ is the specimen cross-section area $\left[\mathrm{cm}^{2}\right]$, and $l$ is the distance between the electrodes [cm].

$\mathrm{Ar}(99.999 \%)$ gas and $\mathrm{O}_{2}$ (99.999\%) gas were used to control the oxygen partial pressures. The measurement range was $10^{-3} \sim 1$ atm; Fig. 2 shows the atmosphere control system and the electrical conductivity measurement equipment.

\subsection{Seebeck Coefficient Measurement}

The Seebeck coefficient was evaluated by measuring the thermoelectric power produced when a temperature gradient was applied to both sides of the material. The equation was given as $\mathrm{S}=\Delta \mathrm{V} / \Delta \mathrm{T}$, where the major charge carrier becomes holes for a positive Seebeck coefficient (S) and the major charge carrier becomes electrons for a negative Seebeck coefficient. The specimen was prepared in rectangular form with dimensions of $2 \times 2 \times 10 \mathrm{~mm}^{3}$. The Ozawa system (RZ2001i, Ozawa, Japan) was used to measure the Seebeck coefficient with varying temperature in an Ar gas atmosphere and in an ambient air condition, respectively.

\section{Results and Discussion}

\subsection{Microstructure Observation and Phase Analysis}

Figure 3 shows SEM (Scanning Electron Microscope) surface images of pure BFO $(x=0,0 B F O)$ and Bi-rich BFO $(x=$ 0.05, 5BFO). Fig. 3(a) shows the microstructure for $0 \mathrm{BFO}$ sintered at $820^{\circ} \mathrm{C}$ using the conventional method; Figs. 3(b) and (c) show the microstructures of $0 \mathrm{BFO}$ and $5 \mathrm{BFO}$, respectively, sintered using the two-step sintering method. The presence of voids within the grains can be observed in Fig. 3(a) for the BFO ceramic fabricated using the conventional sintering process, while the problem of voids appeared to be somewhat improved for 0BFO, fabricated through twostep sintering, as can be observed in Fig. 3(b). Especially, Fig. 3(c) shows that 5BFO, which employed two-step sintering, exhibited a complete disappearance of voids on the surface. The differences in the microstructure were determined to be due to Bi volatility reduction by the two-step sintering and $\mathrm{Bi}$ volatility compensation due to the presence of rich Bi. As can be observed in Fig. 3(c), the effect of Bi excess was significant. Consequently, the relative densities shown in Fig. 3(a), (b), and (c) were 89\%, 92.5\%, and 95.5\%, respectively, which reveal a direct trend in relation to the variation of the open pores.

Figure 4 shows the crystal phase analysis results for $0 \mathrm{BFO}$ and $5 \mathrm{BFO}$, obtained using XRD (X-ray diffractometer). Si powder was used as the standard to compensate for the sample loading height and tilt errors. The diffraction patterns for $0 \mathrm{BFO}$ and $5 \mathrm{BFO}$ revealed rhombohedral crystal structures; $0 B F O$ showed a diffraction pattern similar to that of the single phase, along with Fe-rich secondary phase. 5BFO exhibited a Bi-rich secondary phase and Ferich secondary phase, as was the case for $0 \mathrm{BFO}$.

\subsection{Electrical Conductivity}

$\mathrm{BFO}$ is of $p$-type or $n$-type depending on the addition of

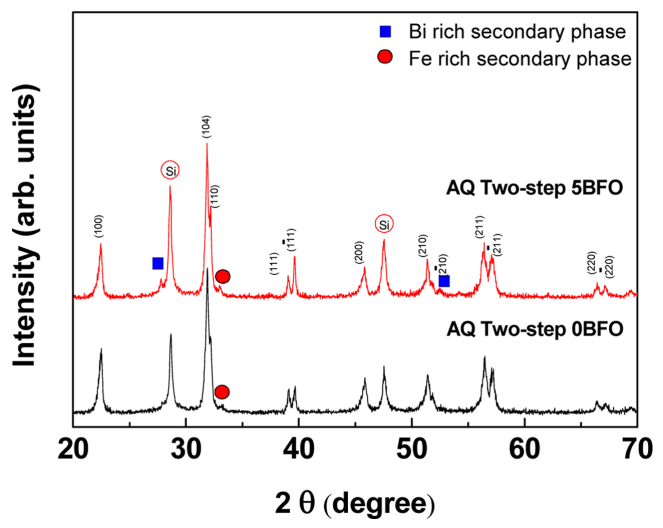

Fig. 4. XRD patterns of air-quenched (AQ) (a) OBFO and (b) 5BFO.
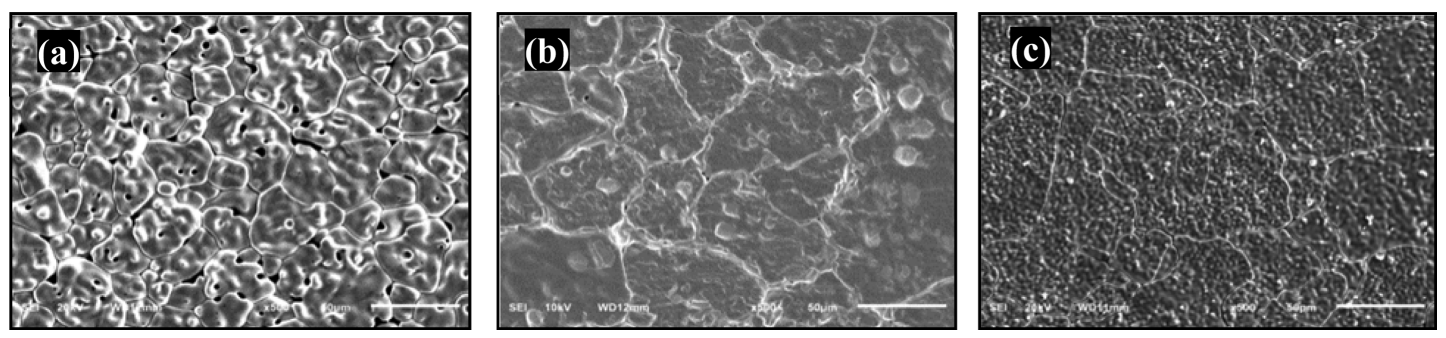

Fig. 3. SEM images of (a) conventionally sintered 0BFO, (b) two-step sintered 0BFO, and (c) two-step sintered 5BFO. 

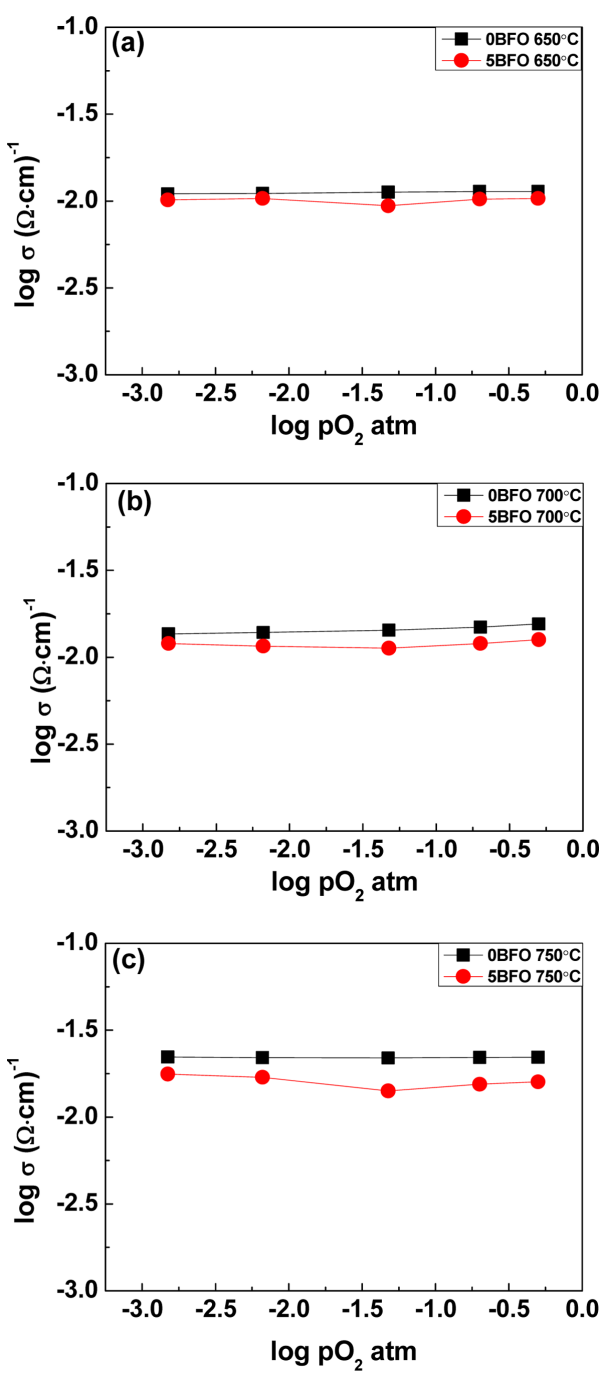

Fig. 5. Electrical conductivity as a function of oxygen partial pressure $\left(\mathrm{pO}_{2}\right)$ for $0 \mathrm{BFO}$ and $5 \mathrm{BFO}$ ceramics at (a) $650^{\circ} \mathrm{C}$, (b) $700^{\circ} \mathrm{C}$, and (c) $750^{\circ} \mathrm{C}$.

various rare earth metals; it shows varying electrical properties. Equivalent impurities that can enter the A-site include $\mathrm{La}^{3+}, \mathrm{Nd}^{3+}$, and $\mathrm{Sm}^{3+}$, while acceptor impurities include $\mathrm{Ca}^{2+}, \mathrm{Sr}^{2+}, \mathrm{Ba}^{2+}$, and $\mathrm{Pb}^{2+}$. Donor impurities that can enter the B-site include $\mathrm{V}^{5+}, \mathrm{Nb}^{5+}, \mathrm{Mn}^{4+}$, and $\mathrm{Ti}^{4+}{ }^{47-20)}$ Additionally, BFO can have oxygen vacancy defects due to $\mathrm{Bi}$ volatility and Fe electrovalence transition. In this study, the electrical properties of pure BFO were investigated.

Figure 5 shows the electrical conductivity measurement results for 0BFO and 5BFO. Fig. 6 provides a diagram of the electrical property based on the defects in BFO. Compared to this, no variation of the electrical conductivity as a function of the oxygen partial pressures $\left(p \mathrm{O}_{2}\right)$ can be observed in Fig. 5(a) - (c). This behavior suggests that the ionic conductivity is higher than the electronic conductivity within the BFO. This can be explained through the following reaction formula.

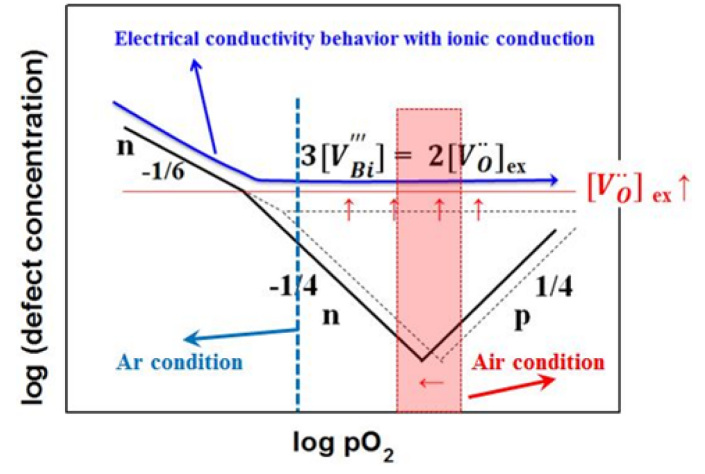

Fig. 6. Schematic diagram of defect concentration (electrical conductivity) vs. oxygen partial pressure $\left(p \mathrm{O}_{2}\right)$.

$$
\begin{aligned}
& 2 \mathrm{BiFeO}_{3} \rightarrow 2 \mathrm{Fe}_{\mathrm{Fe}}^{\mathrm{X}}+3 \mathrm{O}_{\mathrm{O}}^{\mathrm{X}}+2 \mathrm{~V}_{\mathrm{Bi}}^{\prime \prime \prime}+3 \mathrm{~V}_{\mathrm{O}}^{*}+\mathrm{Bi}_{2} \mathrm{O}_{3} \uparrow \\
& 2 \mathrm{FeO} \rightarrow 2 \mathrm{Fe}_{\mathrm{Fe}}^{\prime}+\mathrm{V}_{\mathrm{O}}^{*}+2 \mathrm{O}_{\mathrm{O}}^{\mathrm{X}}+2 \mathrm{~V}_{\mathrm{Bi}}^{\prime \prime \prime}+3 \mathrm{~V}_{\mathrm{O}}^{*}+\mathrm{Bi}_{2} \mathrm{O}_{3} \uparrow \\
& \mathrm{O}_{\mathrm{O}}^{\mathrm{X}} \leftrightarrow \frac{1}{2} \mathrm{O}_{2}(\mathrm{~g})+\mathrm{V}_{\mathrm{O}}^{*}+2 \mathrm{e}^{\prime} \\
& \frac{1}{2} \mathrm{O}_{2}(\mathrm{~g})+\mathrm{V}_{\mathrm{O}}^{*} \leftrightarrow \mathrm{O}_{\mathrm{O}}^{\mathrm{X}}+2 \mathrm{~h}
\end{aligned}
$$

Equation (2) is the reaction formula for Bi volatility; Eq. (3) is the reaction formula for Fe transition, incorporating Eqs. (2) and (4). Thus, it can be explained that the ionic conductivity by oxygen vacancy $\left(\mathrm{V}_{\mathrm{O}}^{*}\right)$ is dominant due to oxygen vacancy compensation for Bi volatility or transition of Fe from $\mathrm{Fe}^{3+}$ to $\mathrm{Fe}^{2+}$. For the latter case of $\mathrm{Fe}$ transition (Eq. (3)), the dominant defect reacts according to Eq. (2) because the latter case is derived from oxygen vacancy due to Bi volatility. Eqs. (4) and (5) are the reaction formula for each of the oxidation and reduction reactions; the above 4 types of defects coexist in a manifold manner. The dominant defect reaction differs according to the degree of oxygen vacancy and the degree of Bi volatility (or Bi content). Figs. 5(a) - (c) show that the electrical conductivity value (log o) for $5 \mathrm{BFO}$ was lower than that for 0BFO. This difference was thought to indicate a decrease in the overall electrical conductivity $\left(\sigma_{\text {total }}=\sigma_{\text {ion }}+\sigma_{\text {electronic }}\right)$ as the ionic conductivity $\left(\sigma_{\text {ion }}\right)$ decreased due to the decreased oxygen vacancy concentration as the Bi volatility amount was compensated for with the $5 \%$ excess $\mathrm{Bi}$ in the sintering process. This type of ionic conductivity phenomenon was in agreement with the results of Yeo et $a l .{ }^{21)}$ but in contrast to the reported $n$-p transition behavior with dominant electron/hole conduction, as reported by Rojac et $a l .{ }^{22}$ This shows that ionic conduction overwhelming electronic conductivity $\left(\sigma_{\text {electronic }}\right)$ can occur sufficiently with $\mathrm{Bi}$ volatility because the electrical conductivity difference is due to the difference in the actual nonstoichiometry in the fabrication of pure BFO. Evidence of the ionic conductivity was also observed in the dielectric loss measurement results, which will be shown later.

Figure 7 shows the activation energy $\left(E_{a}\right)$ for electrical conduction obtained for each specimen on an Arrhenius 

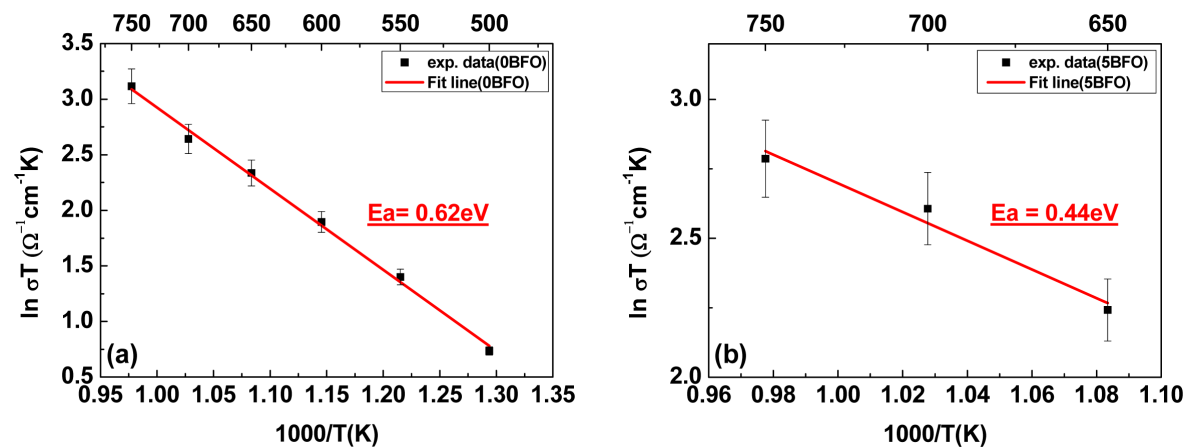

Fig. 7. Arrhenius plots of electrical conductivity for air-quenched (AQ) (a) 0BFO and (b) 5BFO.

plot. The slopes of the Arrhenius plot were used to calculate the activation energy through the below equation.

$$
\ln \sigma \mathrm{T} \propto-\frac{E_{a}}{k_{B} T}
$$

The activation energy values of $0 \mathrm{BFO}$ (Fig. 7(a)) and 5BFO (Fig. 7(b)) were 0.62 and $0.44 \mathrm{eV}$, respectively. These values are within the $0.4-1.0 \mathrm{eV}$ activation energy range for oxygen vacancy movement for typical $\mathrm{ABO}_{3},{ }^{23-25)}$ thus, all the obtained activation energy results showed ionic conductivity behavior due to oxygen vacancy.

\subsection{Seebeck Coefficient}

In order to investigate the types of charge carriers, the Seebeck coefficient of $0 B F O$ was measured as shown in Fig. 8. The measurements were conducted for in air and Ar gas atmospheres and a temperature range of $200-500^{\circ} \mathrm{C}$. The Seebeck coefficient represents the thermoelectric power of the electron/hole carrier rather than that of the ion carrier. As shown in the oxygen partial pressure and electrical conductivity diagram of Fig. 6, the contribution of ionic conductivity was high in the measured oxygen partial pressure regime, and so the overall electrical conductivity was a constant value regardless of the oxygen partial pressure. However, minor carriers with low contribution actually coexist within the material and electron/hole conductivity transition ( $n-p$ transition) behavior was observed for the oxygen partial pressure of this regime. Such $n-p$ transition was also easily observed in the results of Rojac et al. ${ }^{19)}$ which showed dominant electron/hole conductivity due to low oxygen vacancy concentration. In this study, the oxygen vacancy concentration for the measured 0BFO is relatively high, and so the $n-p$ transition regime moves towards the low oxygen partial pressure because the ionic conduction by the oxygen vacancies is dominant. This movement of the $n-p$ transition regime is also observed with temperature decrease. Thus, although the Seebeck coefficient measured in air atmosphere had a positive value ( $p$-type) at low temperature, the Seebeck coefficient became negative or of $n$-type with temperature increase. A decrease in the absolute value of the Seebeck coefficient means that the charge carrier concentration increased. The relationship between the charge carrier

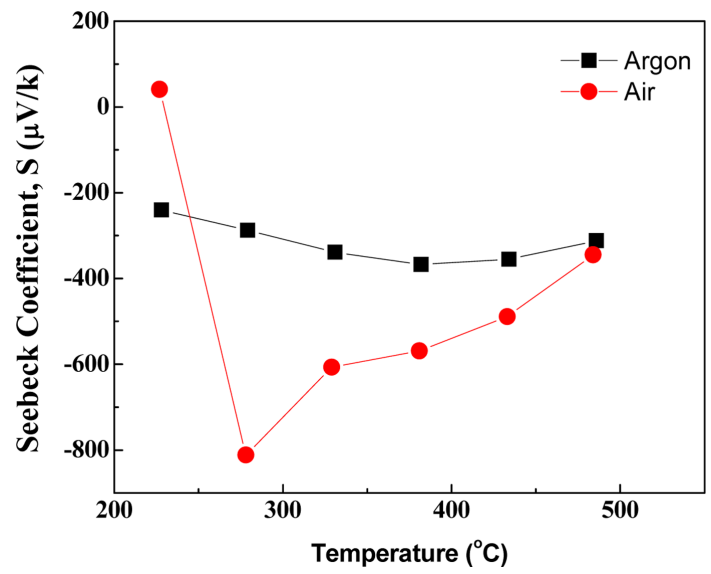

Fig. 8. Seebeck coefficient $(S)$ vs. temperature for $0 \mathrm{BFO}$ in different atmospheres (Argon and air).

concentration $(n)$ and Seebeck coefficient $(S)$ can be expressed by the Pisarenko expression, given in Eq. (7). ${ }^{26)}$

$$
S=\frac{8 \pi^{2} k_{B}^{2}}{3 e h^{2}}\left(\frac{\pi}{3 n}\right)^{2 / 3} m_{d}^{*} T
$$

Here, $k_{B}$ is the Boltzmann constant, $h$ is the Planck constant, and $m_{d}^{*}$ is the density of state (DOS) effective mass. The Seebeck coefficient (S) is inversely proportional to the charge carrier concentration (n). The Seebeck coefficient was a negative value or was of $n$-type for the Ar gas atmosphere. The absolute value of the Seebeck coefficient was smaller than that of the air atmosphere; this signifies the increase of the electron concentration in the Ar gas atmosphere, as shown in Eq. (7) and Fig. 6.

\subsection{P-E Hysteresis Loop}

Figure 9 shows the P-E hysteresis loop measurement results for $0 \mathrm{BFO}$ and $5 \mathrm{BFO}$. The measurement results show that the remnant polarization $\left(P_{\mathrm{r}}\right)$ values for $0 \mathrm{BFO}$ and $5 \mathrm{BFO}$ were $91 \mu \mathrm{C} / \mathrm{cm}^{2}$ and $83 \mu \mathrm{C} / \mathrm{cm}^{2}$, respectively. These values were similar to the typical value of $90-100 \mu \mathrm{C} / \mathrm{cm}^{2}$ for BFO (single crystal). However, it is anticipated that the absence of complete saturation in the P-E hysteresis loop means that the hysteresis loop do not show capacitance but 


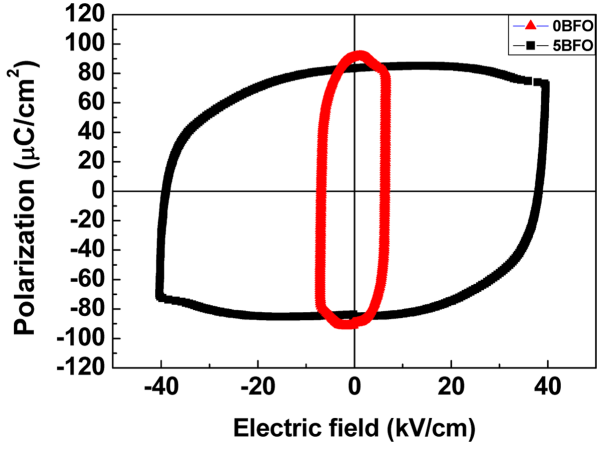

Fig. 9. Room temperature ferroelectric hysteresis $(P-E)$ loops of air-quenched (AQ) $0 \mathrm{BFO}$ and 5BFO.

rather behavior due to current within the material or leakage current amount. The reason behind the low polarization value for $5 \mathrm{BFO}$ was thought to be the decrease in the leakage current compared to that of $0 \mathrm{BFO}$ due to the oxygen vacancy concentration decrease from excess $\mathrm{Bi}$. Observation of the coercive field also revealed that the coercive field of $5 \mathrm{BFO}$ was greater than that of $0 \mathrm{BFO}$. This proves the idea that the dielectric loss of $5 \mathrm{BFO}$ is small.

\subsection{Dielectric Constant and Dielectric Loss Mea- surement}

Figure 10 shows the values of dielectric constant and dielectric loss of $0 \mathrm{BFO}$ and $5 \mathrm{BFO}$. The measured dielectric constants shown in Figs. 10(a) and (c) indicate that the dielectric constant decreased as the measuring frequency (1, $10,100 \mathrm{kHz}$ ) increased and the emerging Neel temperature $\left(\mathrm{T}_{\mathrm{N}}\right)$ range increased as the frequency increased. This frequency dependency clearly shows multiferroicity. The dielectric losses shown in Figs. 10(b) and (d) reveal large dielectric loss values starting from the low temperature range; the dielectric loss value for 0BFO, shown in Fig. 10(b), rapidly increased starting at a temperature lower than was the case for 5BFO, as shown in Fig. 10(d). This shows that dielectric loss occurs easily due to the higher oxygen vacancy concentration, which was compensated for Bi vacancy, of $0 \mathrm{BFO}$ compared to that of $5 \mathrm{BFO}$. The high dielectric loss for BFO at a specific temperature and the lower frequency was in good agreement with the high ionic conductivity in the high temperature electrical conductivity results. This clearly shows that the oxygen vacancy concentration of $\mathrm{BFO}$ is high and transport is also high.

\section{Conclusions}

In this study, the microstructure, electrical conductivity, and $\mathrm{P}-\mathrm{E}$ hysteresis curve were investigated for $\mathrm{X}=(0,0.05)$ $\mathrm{Bi}_{1+\mathrm{x}} \mathrm{FeO}_{3 \pm \delta}$ ceramics at various oxygen partial pressure atmospheres. The $0 \mathrm{BFO}$ and $5 \mathrm{BFO}$ ceramics were synthesized using the solid-state reaction method; to improve the microstructure and porosity, the synthesized ceramics were sintered using the two-step sintering method. High temperature electrical conductivity was measured to assess the main defect behavior of BFO. The high ionic conductivity
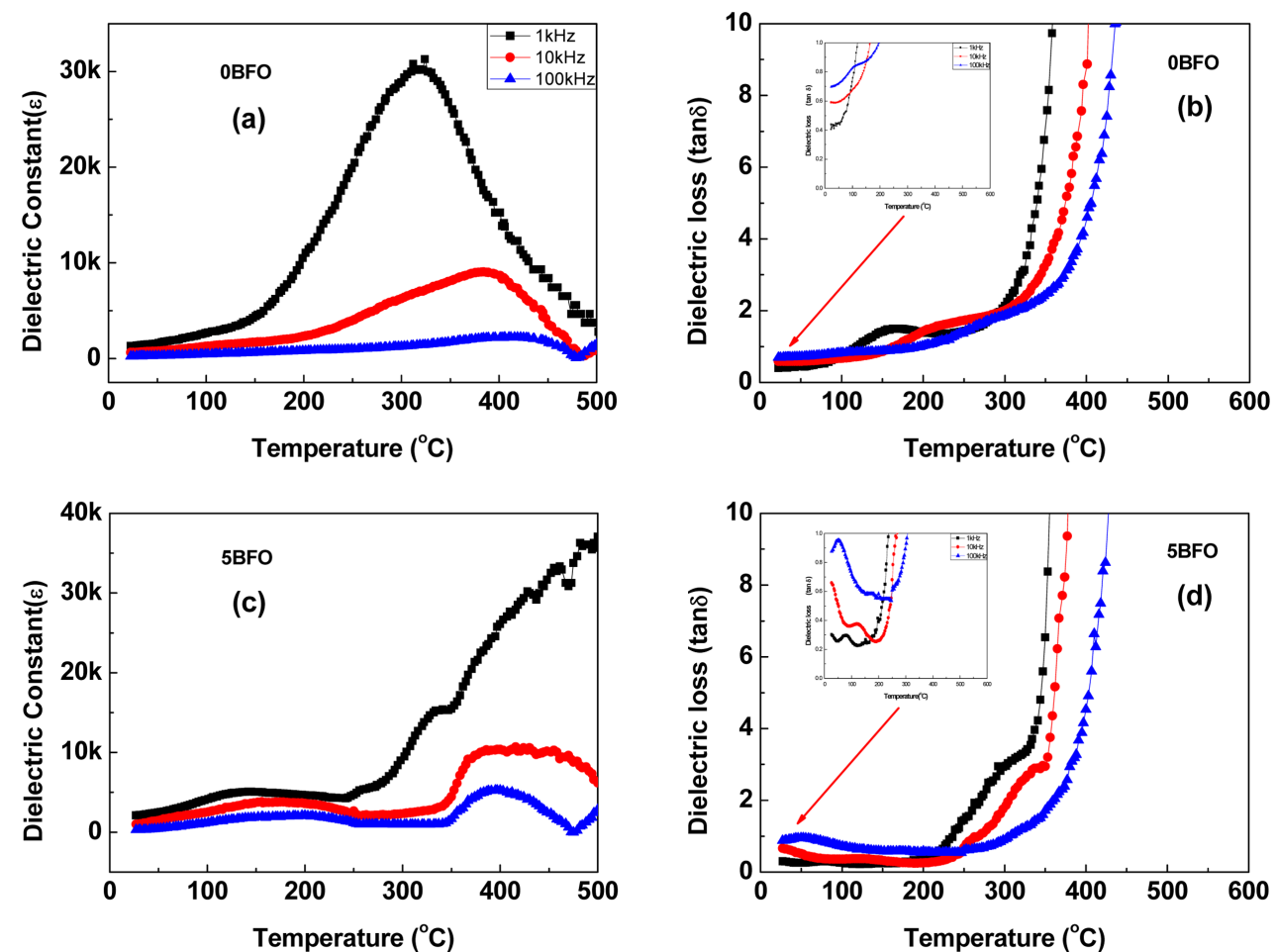

Fig. 10. Temperature dependence of dielectric constant $(\varepsilon)(a, c)$ and dielectric loss $(\tan \delta)(b, d)$ for the air-quenched (AQ) $0 B F O$ and $5 \mathrm{BFO}$ with measurement frequency from $1 \mathrm{kHz}$ to $100 \mathrm{kHz}$. 
due to oxygen vacancy compensation reaction $\left(3\left[V_{\mathrm{Bi}}^{\prime \prime \prime}\right] \approx 2\left[V_{O}^{\prime \prime}\right]\right)$, resulting from the $\mathrm{Bi}$ volatility, and thereby oxygen vacancy compensation reaction $\left(\left[F_{F e}^{\prime}\right] \approx 2\left[V_{O}^{\prime \prime}\right]\right)$ by the Fe electrovalence transition, was identified. With those oxygen vacancies, BFO was found to have a high leakage current. Volatile Bi during sintering was compensated for by excess $\mathrm{Bi}$, and thereby there was a reduction of the oxygen vacancy concentration, which decreased the overall electrical conductivity due to the resulting ionic conductivity decrease. Such ionic conductivity was also observed in the activation energy results of 0.62 and $0.44 \mathrm{eV}$. Although the ionic conductivity is dominant, the minor charge carrier was found to be of $n$-type, with a negative Seebeck coefficient value. The polarization values of $91 \mu \mathrm{C} / \mathrm{cm}^{2}$ and $83 \mu \mathrm{C} / \mathrm{cm}^{2}$ were within the typical BFO range of $90-100 \mu \mathrm{C} / \mathrm{cm}^{2}$, but the P-E hysteresis curve showed high leakage current by oxygen vacancies. Furthermore, the high dielectric loss and its frequency dependence clearly showed the characteristic of leakage current due to oxygen vacancy.

\section{Acknowledgements}

This work was supported by the basic research program through the National Research Foundation of Korea (NRF), funded by Ministry of Education, Science and Technology (MEST) (2011-0030058) and the National Research Foundation of Korea (NRF-2015R1D1A1A01061479).

\section{REFERENCES}

1. M. H. Lee, D. J. Kim, J. S. Park, S. W. Kim, T. K. Song, M.-H. Kim, W.-J. Kim, D. Do, and I.-K. Jeong, "High-Performance Lead-Free Piezoceramics with High Curie Temperatures," Adv. Mater., 27 [43] 6976-82 (2015).

2. K. T. Lee, J. S. Park, J. H. Cho, Y. H. Jeong, J. H. Paik, and J. S. Yun, "The Study on the Phase Transition and Piezoelectric Properties of $\mathrm{Bi}_{0.5}\left(\mathrm{Na}_{0.78} \mathrm{~K}_{0.22}\right)_{0.5} \mathrm{TiO}_{3}-\mathrm{LaMnO}_{3}$ Lead-Free Piezoelectric Ceramics," J. Korean Ceram. Soc., 52 [4] 237-42 (2015).

3. J. Kim, I. Seo, J. Hur, D. Kim, and S. Nahm, "Effect of $\mathrm{MnO}_{2}$ Addition on Microstructure and Piezoelectric Properties of $0.95\left(\mathrm{Na}_{0.5} \mathrm{~K}_{0.5}\right) \mathrm{NbO}_{3}-0.05 \mathrm{CaTiO}_{3}$ Piezoelectric Ceramics," J. Korean Ceram. Soc., 53 [2] 129-33 (2016).

4. H. Han, I. Hong, Y. Kong, J. Lee, and W. Jo, "Effect of Nb Doping on the Dielectric and Strain Properties of Leadfree 0.94 $\left(\mathrm{Bi}_{1 / 2} \mathrm{Na}_{1 / 2}\right) \mathrm{TiO}_{3}-0.06 \mathrm{BaTiO}_{3}$ Ceramics," J. Korean Ceram. Soc., 53 [2] 145-49 (2016).

5. L. W. Martin, S. P. Crane, Y.-H. Chu, M. B. Holcomb, M. Gajek, M. Huijben, N. Balke, and R. Ramesh, "Multiferroics and Magnetoelectrics: Thin Films and Nanostructures," J. Phys.: Condens. Matter., 20 [43] 434220 (2008).

6. X.-Z. Chen, R.-L. Yang, J.-P. Zhou, X.-M. Chen, Q. Jiang, and P. Liu, "Dielectric and Magnetic Properties of Multiferroic $\mathrm{BiFeO}_{3}$ Ceramics Sintered with the Powders Prepared by Hydrothermal Method," Solid State Sci., 19117. 21 (2013)

7. J. B. Neaton, C. Ederer, U. V. Waghmare, N. A. Spaldin, and K. M. Rabe, "First-Principles Study of Spontaneous Polarization in Multiferroic $\mathrm{BiFeO}_{3}$," Phys. Rev. B, 71 [1] 014113 (2005).

8. Z. Zhang, P. Wu, L. Chen, and J. Wang, "Density Functional Theory Plus U Study of Vacancy Formations in Bismuth Ferrite," Appl. Phys. Lett., 96 [23] 232906 (2010).

9. S. Guilin, S. Jian, N. Zhang, and C. Fanggao, "Effects of Oxgen Content on the Electric and Magnetic Properties of $\mathrm{BiFeO}_{3}$ Compound," Phys. B, 493 47-52 (2016).

10. S. M. Selbach, M.-A. Einarsrud, and T. Grande, "On the Thermodynamic Stability of $\mathrm{BiFeO}_{3}$," Chem. Mater., 21 [1] 169-73 (2009).

11. S. M. Selbach, T. Tybell, M.-A. Einarsrud, and T. Grande, "Phase Transitions, Electrical Conductivity and Chemical Stability of $\mathrm{BiFeO}_{3}$ at High Temperatures," J. Solid State Chem., 183 [5] 1205-8 (2010).

12. Z. Dai and Y. Akishige, "Electrical Properties of Multiferroic $\mathrm{BiFeO}_{3}$ Ceramics Synthesized by Spark Plasma Sintering," J. Phys. D: Appl. Phys., 43 [44] 445403 (2010).

13. A. Perejón, N. Masó, A. R. West, P. E. Sánchez-Jiménez, R. Poyato, J. M. Criado, and L. A. Pérez-Maqueda, "Electrical Properties of Stoichiometric $\mathrm{BiFeO}_{3}$ Prepared by Mechanosynthesis with Either Conventional or Spark Plasma Sintering," J. Am. Ceram. Soc., 96 [4] 1220-27 (2013).

14. N. Jeon, K.-S. Moon, D. Rout, and S.-J. L. Kang, "Enhanced Sintering Behavior and Electrical Properties of Single Phase $\mathrm{BiFeO}_{3}$ Prepared by Attrition Milling and Conventional Sintering," J. Korean Ceram. Soc., 49 [6] 485-92 (2012).

15. D. Rout, K.-S. Moon, and S.-J. L. Kang, "TemperatureDependent Raman Scattering Studies of Polycrystalline $\mathrm{BiFeO}_{3}$ Bulk Ceramics," J. Raman Spectrosc., 40 [6] 61826 (2009).

16. R.-Q. Yin, B.-W. Dai, P. Zheng, J.-J. Zhou, W.-F. Bai, F. Wen, J.-X. Deng, L. Zheng, J. Du, and H.-B. Qin, "PurePhase $\mathrm{BiFeO}_{3}$ Ceramics with Enhanced Electrical Properties Prepared by Two-Step Sintering," Ceram. Int., 43 [8] 6467-71 (2017).

17. N. Masó and A. R. West, "Electrical Properties of CaDoped $\mathrm{BiFeO}_{3}$ Ceramics: From $p$-Type Semiconduction to Oxide-Ion Conduction," Chem. Matter., 24 [11] 2127-32 (2012).

18. G. Arya, R. K. Kotnala, and N. S. Negi, "A Novel Approach to Improve Properties of $\mathrm{BiFeO}_{3}$ Nanomultiferroics," $J$. Am. Ceram. Soc., 97 [5] 1475-80 (2014).

19. J. Wei, Y. Liu, X. Bai, C. Li, Y. Liu, Z. Xu, P. Gemeiner, R. Haumont, I. C. Infante, and B. Dkhil, "Crystal Structure, Leakage Conduction Mechanism Evolution and Enhanced Multiferroic Properties in Y-doped $\mathrm{BiFeO}_{3}$ Ceramics," Ceram. Int., 42 [12] 13395-403 (2016).

20. K. Abe, N. Sakai, J. Takahashi, H. Itoh, N. Adachi, and T. Ota, "Leakage Current Properties of Cation-Substituted $\mathrm{BiFeO}_{3}$ Ceramics," Jpn. J. Appl. Phys., 49 [9S] 09MB01 (2010).

21. H.-G. Yeo, T.-K. Song, Y.-S. Sung, J.-H. Cho, H.-M. Lee, and M.-H. Kim, "Effects of Nb Substitution on the Leakage Currents and the Electrical Properties of Ceramics," New Phys.: Sae Mulli., 56 [3] 278-82 (2008). 
22. T. Rojac, A. Bencan, B. Malic, G. Tutuncu, J. L. Jones, J. E. Daniels, and D. Damjanovic, " $\mathrm{BiFeO}_{3}$ Ceramics: Processing, Electrical, Electromechanical Properties," J. Am. Ceram. Soc., 97 [7] 1993-2011 (2014).

23. S. J. Clacrk and J. Robertson, "Energy Levels of Oxygen Vacancies in $\mathrm{BiFeO}_{3}$ by Screened Exchange," Appl. Phys. Lett., 94 [2] 022902 (2009).

24. H.-Y. Su and K. Sun, "DFT Study of Stability of Oxygen
Vacancy in Cubic $\mathrm{ABO}_{3}$ Perovskites," J. Mater. Sci., 50 [4] 1701-9 (2015)

25. W. L. Warren, K. Vanheusden, D. Dimos, G. E. Pike, and B. A. Tuttle, "Oxygen Vacancy Motion in Perovskite Oxides," J. Am. Ceram. Soc., 79 [2] 536-38 (1996).

26. G. J. Snyder and E. S. Toberer, "Complex Thermoelectric Materials," Nat. Mater., 7 [2] 105-14 (2008). 